\title{
Tychonoff Products of Two-Element Sets and Some Weakenings of the Boolean Prime Ideal Theorem
}

\author{
by \\ Kyriakos KEREMEDIS \\ Presented by Czestaw RYLL-NARDZEWSKI
}

Summary. Let $X$ be an infinite set, and $\mathcal{P}(X)$ the Boolean algebra of subsets of $X$. We consider the following statements:

$\operatorname{BPI}(X)$ : Every proper filter of $\mathcal{P}(X)$ can be extended to an ultrafilter.

$\mathrm{UF}(X): \mathcal{P}(X)$ has a free ultrafilter.

We will show in ZF (i.e., Zermelo-Fraenkel set theory without the Axiom of Choice) that the following four statements are equivalent:

(i) $\operatorname{BPI}(\omega)$.

(ii) The Tychonoff product $2^{\mathbb{R}}$, where 2 is the discrete space $\{0,1\}$, is compact.

(iii) The Tychonoff product $[0,1]^{\mathbb{R}}$ is compact.

(iv) In a Boolean algebra of size $\leq|\mathbb{R}|$ every filter can be extended to an ultrafilter.

We will also show that in $\mathrm{ZF}, \mathrm{UF}(\mathbb{R})$ does not imply $\mathrm{BPI}(\mathbb{R})$. Hence, $\mathrm{BPI}(\mathbb{R})$ is strictly stronger than $\operatorname{UF}(\mathbb{R})$. We do not know if $\operatorname{UF}(\omega)$ implies $\operatorname{BPI}(\omega)$ in $\mathrm{ZF}$.

Furthermore, we will prove that the axiom of choice for sets of subsets of $\mathbb{R}$ does not imply $\operatorname{BPI}(\mathbb{R})$ and, in addition, the axiom of choice for well orderable sets of non-empty sets does not imply BPI $(\omega)$.

1. Notation and terminology. Let $X$ be a non-empty set. We say that a family $\mathcal{H} \subset \mathcal{P}(X) \backslash\{\emptyset\}$ has the finite intersection property, fip for abbreviation, iff $\bigcap Q \neq \emptyset$ for every finite subset $Q$ of $\mathcal{H}$.

It is well known that there are many characterizations of the notion of "compact", all equivalent in ZFC set theory (i.e., ZF plus the Axiom of Choice). However, in the absence of the Axiom of Choice these "types" of compactness may fail to be equivalent. Consequently, the notion of a com-

2000 Mathematics Subject Classification: 03G05, 03E25, 54B10, 54D30.

Key words and phrases: Boolean algebra, prime ideal, filter, ultrafilter, Axiom of Choice, weak axioms of choice, Tychonoff products. 
pact topological space does not have a clear meaning in ZF set theory. In this paper we shall be using the Heine-Borel definition of compactness, i.e., a topological space $(X, T)$ is compact iff every open cover of $X$ has a finite subcover. Equivalently, $X$ is compact iff every family of closed subsets of $X$ having the fip has a non-empty intersection. For the interrelation between several definitions of compactness in ZF, the reader is referred to [1], [2], [6], [7].

We will consider the following statements:

1. BPI: Every Boolean algebra has a prime ideal. \{It is known that BPI is equivalent in $\mathrm{ZF}$ to the statement that every Boolean algebra has an ultrafilter and also to the statement (for every $X) \operatorname{BPI}(X)$; see [8].\}

2. UF: For every infinite set $X, \mathcal{P}(X)$ has a free ultrafilter (i.e., (for every infinite set $X) \mathrm{UF}(X))$.

3. $\mathrm{AC}(\mathbb{R})$ : Every family of non-empty subsets of $\mathbb{R}$ has a choice function.

4. $\mathrm{CAC}(\mathbb{R}): \mathrm{AC}(\mathbb{R})$ restricted to countable families.

5. $\mathrm{PW}(\mathbb{R})$ : The powerset $\mathcal{P}(\mathbb{R})$ of $\mathbb{R}$ is well orderable.

6. IDI: Every infinite set is Dedekind infinite (i.e., it has a countably infinite subset).

7. WOAC: Every well orderable set of non-empty sets has a choice function.

8. $\operatorname{TP}\left(2^{\mathbb{R}}\right)$ : The Tychonoff product $2^{\mathbb{R}}$, where 2 is the discrete space $\{0,1\}$, is compact.

9. DC, the axiom of dependent choices: If $R$ is a non-empty relation on a non-empty set $X$ such that $(\forall x)(\exists y)(x R y)$, then there exists a function $f: \omega \rightarrow X$ such that $f(n) R f(n+1)$ for all $n \in \omega$.

The reader is referred to [5] for notions on Boolean algebras which are used but not defined in this paper.

Note. Most of the propositions which are listed above are discussed in the book [8] on the consequences of the Axiom of Choice by P. Howard and J. E. Rubin as "Form x", "x" being some numeral. For example, in [8], the Boolean Prime Ideal Theorem BPI is named "Form 14". The authors [8] have developed software available at http://www.math.purdue.edu/ jer/Papers/conseq.html where one can insert Form numerals to retrieve all known provable or refutable implications between these Forms. For the reader's convenience we give the Form numerals of [8] for those propositions which can be found in the latter book:

BPI (Form 14), BPI $(\omega)$ (Form 225), UF (Form 63), UF $(\omega)$ (Form 70), $\mathrm{AC}(\mathbb{R})($ Form 79$), \mathrm{CAC}(\mathbb{R})($ Form 94$), \mathrm{PW}(\mathbb{R})$ (Form 130), IDI (Form 9), WOAC (Form 40), TP $\left(2^{\mathbb{R}}\right)$ (Form 139), DC (Form 43).

2. Introduction and some preliminary results. The main purpose of this paper is to give six answers to questions in the table below (which 
were not given in [8]). In this table if the entry in row A and column B is:

- "?", then nothing is known about the implication $\mathrm{A} \rightarrow \mathrm{B}$ in $\mathrm{ZF}$;

- " $\rightarrow$ ", then $\mathrm{A} \rightarrow \mathrm{B}$ in $\mathrm{ZF}$;

- "†", then $\mathrm{A} \nrightarrow \mathrm{B}$ in $\mathrm{ZF}$.

\begin{tabular}{|c|c|c|c|c|c|c|c|}
\hline & $\operatorname{BPI}(\omega)$ & $\mathrm{UF}(\omega)$ & $A C(\mathbb{R})$ & $\mathrm{CAC}(\mathbb{R})$ & WOAC & $\mathrm{TP}\left(2^{\mathbb{R}}\right)$ & $\mathrm{DC}$ \\
\hline $\operatorname{BPI}(\omega)$ & $\rightarrow$ & $\rightarrow$ & $\not \rightarrow$ & $\nrightarrow$ & $\not \rightarrow$ & $\rightarrow$ & $\not \rightarrow$ \\
\hline $\mathrm{UF}(\omega)$ & $?$ & $\rightarrow$ & $\not \rightarrow$ & $\not \rightarrow$ & $\not \rightarrow$ & $?$ & th \\
\hline $\mathrm{AC}(\mathbb{R})$ & $\rightarrow$ & $\rightarrow$ & $\rightarrow$ & $\rightarrow$ & $\not \rightarrow$ & $\rightarrow$ & $\not \rightarrow$ \\
\hline $\mathrm{CAC}(\mathbb{R})$ & $\not \rightarrow$ & $\not \rightarrow$ & $\not$ & $\rightarrow$ & t & $\not \rightarrow$ & $\not \rightarrow$ \\
\hline WOAC & $\not \rightarrow$ & $\not \rightarrow$ & $\not \rightarrow$ & $\rightarrow$ & $\rightarrow$ & $\not \rightarrow$ & $\rightarrow$ \\
\hline $\operatorname{TP}\left(2^{\mathbb{R}}\right)$ & $\rightarrow$ & $\rightarrow$ & $\not$ & 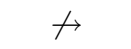 & t & $\rightarrow$ & $\not$ \\
\hline DC & $\not \rightarrow$ & $\not \rightarrow$ & $\not \rightarrow$ & $\rightarrow$ & t & $\not \rightarrow$ & $\rightarrow$ \\
\hline
\end{tabular}

The entries of the table which are established in this paper are the following: $\operatorname{BPI}(\omega) \rightarrow \mathrm{TP}\left(2^{\mathbb{R}}\right), \mathrm{AC}(\mathbb{R}) \rightarrow \mathrm{BPI}(\omega)$ (this implication is not listed in [8] but it is well known and added here for completeness), $\mathrm{CAC}(\mathbb{R}) \nrightarrow$ $\mathrm{TP}\left(2^{\mathbb{R}}\right)$, WOAC $\nrightarrow \mathrm{TP}\left(2^{\mathbb{R}}\right), \mathrm{TP}\left(2^{\mathbb{R}}\right) \rightarrow \mathrm{BPI}(\omega), \mathrm{TP}\left(2^{\mathbb{R}}\right) \rightarrow \mathrm{UF}(\omega), \mathrm{DC} \nrightarrow$ $\mathrm{TP}\left(2^{\mathbb{R}}\right)$. For the positive and independence results for the rest of the entries of the above table, the reader is referred to P. Howard and J. E. Rubin's book [8] and to its webpage.

It was shown in [14] and independently in [18] that BPI is equivalent to Tychonoff's Product Theorem for Hausdorff $\left(T_{2}\right)$ spaces:

$\mathrm{TPT}_{2}$ : The Tychonoff product of compact $T_{2}$ spaces is compact.

J. Mycielski [15] proved that BPI is equivalent to the statement:

$\mathcal{S}$ : For every set $X$ the Tychonoff product $2^{X}$, where 2 has the discrete topology, is compact.

It is part of the folklore (see [10]) that for a well ordered cardinal $m$, the statement "the Tychonoff product $2^{m}$, where 2 has the discrete topology, is compact" is deducible in ZF. J. Truss in analogy with statement $\mathcal{S}$ (see [19]) introduced the statement $\operatorname{TP}\left(2^{\mathbb{R}}\right)$ and in [8] it was asked whether $\operatorname{TP}\left(2^{\mathbb{R}}\right)$ is deducible in $\mathrm{ZF}^{0}$ (i.e., $\mathrm{ZF}$ without the axiom of regularity). In [10] it was shown that $\operatorname{TP}\left(2^{\mathbb{R}}\right)$ fails in Cohen's Second Model (see Model $\mathcal{M} 7$ in $[8])$. However, the status of the implications $\mathrm{CAC}(\mathbb{R}) \rightarrow \mathrm{TP}\left(2^{\mathbb{R}}\right)$, WOAC $\rightarrow$ $\mathrm{TP}\left(2^{\mathbb{R}}\right)$, and $\mathrm{DC} \rightarrow \mathrm{TP}\left(2^{\mathbb{R}}\right)$ remained unknown.

Clearly, BPI implies $\mathrm{TP}\left(2^{\mathbb{R}}\right)$ because 2 with the discrete topology is a compact $T_{2}$ space. Since BPI holds but $\mathrm{CAC}(\mathbb{R})$ fails in Cohen's basic model (see Model $\mathcal{M} 1$ in [8]) it follows that $\mathrm{TP}\left(2^{\mathbb{R}}\right)$ does not imply $\mathrm{CAC}(\mathbb{R})$. In Theorem 8 we show that WOAC, hence $\mathrm{DC}$ and $\mathrm{CAC}(\mathbb{R})$, do not imply $\operatorname{TP}\left(2^{\mathbb{R}}\right)$. Hence $\mathrm{CAC}(\mathbb{R})$ and $\operatorname{TP}\left(2^{\mathbb{R}}\right)$ are independent of each other. 
In [8], $\operatorname{TP}\left(2^{\mathbb{R}}\right)$ and $\operatorname{BPI}(\omega)$ are treated as different statements. However, the equivalence $\mathrm{BPI} \leftrightarrow \mathcal{S}$ is an indication that $\operatorname{TP}\left(2^{\mathbb{R}}\right)$ and $\operatorname{BPI}(\omega)$ express the same truth. We show in Theorem 6 that this is indeed the case.

The propositions UF and UF $(\omega)$ were introduced in [9, Prob. 8.5, p. 172, and Prob. 5.24, p. 82, respectively], and $\operatorname{BPI}(\omega)$ was introduced by Y. Rav (see [16]).

Clearly, $\mathrm{BPI} \rightarrow \mathrm{BPI}(\omega) \rightarrow \mathrm{UF}(\omega)$ and $\mathrm{BPI} \rightarrow \mathrm{UF} \rightarrow \mathrm{UF}(\omega)$, and it is known (see the table of implications at http://www.math.purdue.edu/ $\sim$ jer/Papers/conseq.html) that $\mathrm{UF}(\omega) \nrightarrow \mathrm{UF}$ and $\mathrm{BPI}(\omega) \nrightarrow \mathrm{BPI}$.

In contrast with the status of $\operatorname{UF}(\omega) \rightarrow \operatorname{BPI}(\omega)$, we show in Theorem 10 that $\mathrm{UF}(\mathbb{R}) \nrightarrow \mathrm{BPI}(\mathbb{R})$.

Theorem 1. UF $(\omega)+\mathrm{IDI} \rightarrow \mathrm{UF}$. In particular, $\mathrm{UF}(\omega) \rightarrow \mathrm{UF}(\mathbb{R})$.

Proof. Fix an infinite set $A$ and let, by IDI, $X$ be a countably infinite subset of $A$. Fix, by $\operatorname{UF}(\omega)$, a free ultrafilter $\mathcal{H}$ of $\mathcal{P}(X)$ and let $\mathcal{F}=\{Y \in$ $\mathcal{P}(A): Y$ includes a member of $\mathcal{H}\}$. We show that $\mathcal{F}$ is an ultrafilter of $\mathcal{P}(A)$. To this end, it suffices to show that $\mathcal{F}$ is maximal with respect to the fip. Fix $B \subset A$ such that $\mathcal{F} \cup\{B\}$ has the fip. Fix $Y \in \mathcal{H}$. Then $Z=B \cap Y$ meets non-trivially each member of $\mathcal{H}$. Thus, $Z \in \mathcal{H}$ and consequently $B \in \mathcal{F}$ and $\mathcal{F}$ is maximal as required.

The second assertion follows trivially because $\omega \subset \mathbb{R}$.

REMARK 2. It is known that in Cohen's basic model $\mathcal{M} 1$ in [8], UF holds but IDI fails. Thus, the first implication in Theorem 1 is not reversible. We do not know whether the second one is. We conjecture that it is not.

THEOREM 3.

(i) $\mathrm{PW}(\mathbb{R}) \rightarrow \mathrm{BPI}(\mathbb{R}) \rightarrow \mathrm{BPI}(\omega) \rightarrow \mathrm{UF}(\omega) \rightarrow U F(\mathbb{R})$.

(ii) $\mathrm{AC}(\mathbb{R})$ implies $\mathrm{BPI}(\omega)$.

Proof. (i) $\mathrm{PW}(\mathbb{R}) \rightarrow \operatorname{BPI}(\mathbb{R})$. Fix a filter $\mathcal{H}$ of $\mathcal{P}(\mathbb{R})$ and let $\left\{X_{i}: i \in \aleph\right\}$, where $\aleph$ is a well-ordered cardinal, be a well-ordering of $\mathcal{P}(\mathbb{R})$. Via a straightforward induction on $\aleph, \mathcal{H}$ can be extended to an ultrafilter $\mathcal{F}$ of $\mathcal{P}(\mathbb{R})$.

$\mathrm{BPI}(\mathbb{R}) \rightarrow \mathrm{BPI}(\omega) \rightarrow \mathrm{UF}(\omega)$. These are straightforward.

(ii) This can also be proved inductively.

TheOREM 4 ([20, Theorem 16.4(c)]). In ZFC, a product of Hausdorff spaces with at least two points each is separable iff each factor is separable and there are $\leq|\mathbb{R}|$ factors. In particular, if $(X, T)$ is a separable Hausdorff space then, in $Z F$, the product $X^{\mathbb{R}}$ is separable.

3. Positive results. In [13], Azriel Lévy proved that BPI, hence its equivalent (see [15]) statement: 
$\mathcal{S}$ : For every set $X$ the Tychonoff product $2^{X}$, where 2 has the discrete topology, is compact,

implies the axiom of choice for families of non-empty finite sets, hence it implies the weaker statement:

$\mathcal{C}$ : Every family of two-element sets has a choice function.

We will show similar propositions in the next theorem.

THEOREM 5.

(i) $\operatorname{TP}\left(2^{\mathbb{R}}\right)$ implies that every disjoint family of non-empty sets of reals is linearly orderable.

(ii) $\operatorname{TP}\left(2^{\mathbb{R}}\right)$ implies that every family $\mathcal{A}=\left\{A_{i}: i \in I\right\}$ of non-empty finite subsets of $\mathcal{P}(\mathbb{R})$ such that $\bigcup \mathcal{A}$ is disjoint has a choice function.

(iii) $\operatorname{TP}\left(2^{\mathbb{R}}\right)$ implies that for all $n \in \omega \backslash 2$, every family $\mathcal{A}=\left\{A_{i}: i \in \mathbb{R}\right\}$ of $\leq n$-element subsets of $\mathcal{P}(\mathbb{R})$ has a choice function.

Proof. (i) Let $\mathcal{A}$ be a disjoint family of non-empty sets of reals. Let $\mathcal{L}$ be a propositional language with propositional variables $p_{x y}, x, y \in \mathbb{R}$, which have intended meaning $(x \in A \in \mathcal{A}) \wedge(y \in B \in \mathcal{A})$. Let $P$ be the set of all propositions of the language $\mathcal{L}$, and $\Sigma$ be the subset of $P$ consisting of the following four types of propositions:

(1) $p_{x y} \wedge p_{y x}$ for every $A \in \mathcal{A}$ and all $x$ and $y$ in $A$,

(2) $\left(\neg p_{x y}\right) \vee\left(\neg p_{y x}\right)$ for all $A, B \in \mathcal{A}$ with $A \neq B$ and all $x \in A$ and $y \in B$,

(3) $p_{x y} \wedge p_{y z} \rightarrow p_{x z}$ for all $x, y, z \in \mathbb{R}$,

(4) $p_{x y} \vee p_{y x}$ for all $x, y \in \mathbb{R}$.

Clearly, $|P|=\left|2^{\aleph_{0}}\right|$. We seek for a valuation mapping $\sigma \in 2^{P}$ such that $\sigma(\phi)=1$ for all $\phi \in \Sigma$. Then the required linear ordering on $\mathcal{A}$ will be

$$
\mathcal{R}=\left\{(A, B) \in \mathcal{A}^{2}:(\forall x \in A)(\forall y \in B)\left(\sigma\left(p_{x y}\right)=1\right)\right\} .
$$

$\operatorname{By} \operatorname{TP}\left(2^{\mathbb{R}}\right)$ the Tychonoff product $2^{P}$ is compact. For each finite subset $X$ of $\Sigma$ let $C_{X}=\left\{f \in 2^{P}: f\right.$ is a valuation satisfying $\left.X\right\}$. It is easy to verify that $\mathcal{C}=\left\{C_{X}: X \in[\Sigma]^{<\omega}\right\}$ is a family of non-empty closed subsets of $2^{P}$ with the finite intersection property. Hence, there exists a valuation $\sigma \in \bigcap \mathcal{C}$. Clearly, $\sigma$ satisfies $\Sigma$, finishing the proof of (i).

(ii) This follows immediately from (i).

(iii) The proof is by induction on $n \in \omega \backslash 2$.

For $n=2$, fix a family $\mathcal{A}=\left\{A_{i}: i \in \mathbb{R}\right\}$ of two-element subsets of $\mathcal{P}(\mathbb{R})$. For every $i \in \mathbb{R}$ we may assume that the elements of $A_{i}$ are incomparable under the relation $\subset$. (Otherwise, we may choose the element $\bigcap A_{i}$ of $A_{i}$.) It follows that if $a, b$ are the elements of $A_{i}$, then $a \backslash b \neq \emptyset$ and $b \backslash a \neq \emptyset$. Thus, without loss of generality we may assume that for every $i \in \mathbb{R}$ the 
members of $A_{i}$ are disjoint. For every $i \in \mathbb{R}$, let $h_{i}: \mathbb{R} \rightarrow \mathbb{R} \times \mathbb{R}$ be the function given by $h_{i}(x)=(i, x)$ for all $x \in \mathbb{R}$. Fix a $1: 1$ and onto function $f: \mathbb{R} \times \mathbb{R} \rightarrow \mathbb{R}$ (in ZF, $|\mathbb{R} \times \mathbb{R}|=|\mathbb{R}|$ ) and let $f_{i}: \mathbb{R} \rightarrow \mathbb{R}$ be the function given by $f_{i}=f \circ h_{i}$. Clearly, $\mathcal{B}=\left\{f_{i}\left[A_{i}\right]: i \in \mathbb{R}\right\}$ is a family as in (i) of the present theorem and any choice function of the family $\mathcal{B}$ yields a choice function of the family $\mathcal{A}$.

Assume that the result holds for $n=k, k>2$. Fix a family $\mathcal{A}=\left\{A_{i}\right.$ : $i \in \mathbb{R}\}$ of $(k+1)$-element subsets of $\mathcal{P}(\mathbb{R})$. Without loss of generality we may assume that for every $i \in \mathbb{R}$ and any $A, B \in A_{i}, A \backslash B \neq \emptyset$ and $B \backslash A \neq \emptyset$. (Otherwise replace $A_{i}$ by $A_{i}^{*}=\left\{A \in A_{i}: A\right.$ is maximal with respect to inclusion $\}$.) Under the previous assumption we may also infer that $\bigcap A_{i}=\emptyset$ for all $i \in \mathbb{R}$. (Working with $A_{i}^{*}$ instead of $A_{i}$ we find that $\bigcap\left\{\left(\bigcup A_{i}^{*}\right) \backslash A\right.$ : $\left.A \in A_{i}^{*}\right\}=\emptyset$.) Fix an $i \in \mathbb{R}$. For every $x \in \bigcup A_{i}$ we define the degree of $x$ by $\operatorname{deg}(x)=\left|\left\{A \in A_{i}: x \in A\right\}\right|$. Let $n_{i}=\min \left\{\operatorname{deg}(x): x \in \bigcup A_{i}\right\}$ and $B_{i}=\left\{x \in \bigcup A_{i}: \operatorname{deg}(x)=n_{i}\right\}$. Define a binary relation $\sim_{i}$ on $B_{i}$ by requiring for all $x, y \in B_{i}$,

$$
x \sim_{i} y \quad \text { iff } \quad\left(\forall A \in A_{i}\right)(x \in A \leftrightarrow y \in A) .
$$

It can be easily verified that $\sim_{i}$ is an equivalence relation on $B_{i}$ (the symmetry follows from the fact that the elements of $B_{i}$ have the same degree). Let $C_{i}=\left\{[x]_{\sim_{i}}: x \in B_{i}\right\}$. Clearly, $C_{i}$ is a disjoint finite subset of $\mathcal{P}(\mathbb{R})$. Put $\mathcal{C}=\left\{C_{i}: i \in \mathbb{R}\right\}$. As in the case $n=2$ we may assume that $\bigcup \mathcal{C}$ is a disjoint set, hence we may view $\mathcal{C}$ as a family in (ii) of the present theorem. By $\operatorname{TP}\left(2^{\mathbb{R}}\right)$ let $f$ be a choice function for $\mathcal{C}$. For every $i \in \mathbb{R}$, let $A_{i}^{1}=\left\{A \in A_{i}: A \cap f\left(C_{i}\right) \neq \emptyset\right\}$. Then $A_{i}^{1}$ is a proper subset of $A_{i}$ for all $i \in \mathbb{R}$. (If $f\left(C_{i}\right)=\left[x^{*}\right]_{\sim_{i}}$, then since $\bigcap A_{i}=\emptyset$, it follows that $x^{*} \notin A$ for some $A \in A_{i}$. By the definition of $\sim_{i}$ we have $\left[x^{*}\right]_{\sim_{i}} \subset\left(\bigcup A_{i}\right) \backslash A$.) By the induction hypothesis, the family $\mathcal{A}^{1}=\left\{A_{i}^{1}: i \in \mathbb{R}\right\}$ has a choice function. Thus, $\mathcal{A}$ has a choice function and the induction terminates. This completes the proof of (iii) and of the theorem.

THEOREM 6 . The following statements are equivalent:

(i) In a Boolean algebra $\mathcal{B}$ of size $\leq|\mathbb{R}|$ every filter can be extended to an ultrafilter.

(ii) $\operatorname{BPI}(\omega)$.

(iii) For every separable compact $T_{2}$ space $(X, T)$ the product $X^{\mathbb{R}}$ is compact.

(iv) The product $[0,1]^{\mathbb{R}}$ is compact.

(v) Tychonoff products of finite subspaces of $\mathbb{R}$ are compact.

(vi) $\mathrm{TP}\left(2^{\mathbb{R}}\right)$.

(vii) For every propositional language $\mathcal{L}$ of size $\leq|\mathbb{R}|$ and every consistent subset $\Sigma$ of $\mathcal{L}$ there exists a valuation mapping which satisfies $\Sigma$. 
Proof. (i) $\rightarrow$ (ii). This follows from the observation that $(\mathcal{P}(\omega), \triangle, \cap)$ is a Boolean algebra.

(ii) $\rightarrow$ (iii). Fix a separable compact $T_{2}$ space $(X, T)$ and by Theorem 4 , let $D=\left\{d_{n}: n \in \omega\right\}$ be a countable dense subset of $X^{\mathbb{R}}$. We show that the product $X^{\mathbb{R}}$ is compact. Assume by way of contradiction that $\mathcal{U}$ is an open cover of $X^{\mathbb{R}}$ such that no finite subfamily of $\mathcal{U}$ has dense union in $X$. (Since $X$ is compact and $T_{2}$ it follows, in ZF, that $X$ is $T_{3}$. Furthermore, the proof of Theorem 14.4 of [20] uses no choice. Hence, $X^{\mathbb{R}}$ is $T_{3}$. Moreover, it is easy to see that in $\mathrm{ZF}$ a regular space $(X, T)$ is compact iff every open cover of $X$ has a finite subfamily whose union is dense in $X$.) Let $\mathcal{F}$ be an ultrafilter of $\mathcal{P}(D)$ extending the filter $\mathcal{H}$ generated by the family $\mathcal{G}=\left\{U^{\mathrm{c}} \cap D: U \in \mathcal{U}\right\}$. Clearly, for every $i \in \mathbb{R}, \mathcal{F}_{i}=\left\{\pi_{i}[F]: F \in \mathcal{F}\right\}$ is an ultrafilter of $\mathcal{P}\left(\pi_{i}[D]\right)$. Since $X$ is compact and $T_{2}$, and $\mathcal{F}_{i}$ is an ultrafilter of $\mathcal{P}\left(\pi_{i}[D]\right)$, it follows that $\bigcap\left\{\overline{\pi_{i}[F]}: F \in \mathcal{F}\right\}$ is a singleton, say $\left\{x_{i}\right\}$. It is straightforward to verify that the element $x \in X^{\mathbb{R}}$ such that for all $i \in \mathbb{R}, x(i)=x_{i}$, is in $\bigcap\left\{U^{\mathrm{c}}: U \in \mathcal{U}\right\}$. Hence, $\mathcal{U}$ is not a cover of $X^{\mathbb{R}}$ and we have arrived at a contradiction.

(iii) $\rightarrow$ (iv). This is straightforward.

(iv) $\rightarrow(\mathrm{v})$. Fix a family $\left\{X_{i}: i \in I\right\}$ of finite subsets of $\mathbb{R}$. Since $\left|[\mathbb{R}]^{<\omega}\right|=$ $|\mathbb{R}|$ in $\mathrm{ZF}$, without loss of generality we may assume that $|I|=|\mathbb{R}|$. Since $|[0,1]|=|\mathbb{R}|$, we may assume that $X_{i} \subset[0,1]$ for all $i \in \mathbb{R}$. The conclusion now follows from our hypothesis and the fact that the product $X=\prod_{i \in \mathbb{R}} X_{i}$ is a closed subspace of the compact $T_{2}$ space $[0,1]^{\mathbb{R}}$.

$(\mathrm{v}) \rightarrow(\mathrm{vi})$. This is straightforward.

(vi) $\rightarrow$ (vii). Let $\mathcal{L}$ be a propositional language having a continuum-sized set $P$ of propositions, and let $\Sigma$ be a consistent subset of $P$ (i.e. for every finite subset $\Pi$ of $\Sigma$ there is a valuation which satisfies $\Pi)$. By $\operatorname{TP}\left(2^{\mathbb{R}}\right)$ the Tychonoff product $2^{P}$ is compact. For each finite subset $X$ of $\Sigma$ let $C_{X}=$ $\left\{f \in 2^{P}: f\right.$ is a valuation satisfying $\left.X\right\}$. Clearly, $\mathcal{C}=\left\{C_{X}: X \in[\Sigma]^{<\omega}\right\}$ is a family of non-empty closed subsets of $2^{P}$ having the finite intersection property. Hence, there exists a valuation $\sigma \in \bigcap \mathcal{C}$. It follows that $\sigma$ satisfies $\Sigma$ as required.

$($ vii $) \rightarrow($ i). Fix a Boolean algebra $(\mathcal{B},+, \cdot, 1)$ of size $\leq|\mathbb{R}|$ and let $H$ be a filter in $\mathcal{B}$. Let $\mathcal{L}$ be a propositional language with propositional variables $p_{b}, b \in \mathcal{B}$. Here, the propositional variable $p_{b}$ is intended to assert that $b$ lies in the desired ultrafilter.

Let $P$ be the set of all propositions of the language $\mathcal{L}$ and let $\Sigma$ be the set of propositions of the following types:

(1) $p_{b}$ for all $b \in H$,

(2) $p_{b} \rightarrow p_{a}$ for all $a, b \in \mathcal{B}$ such that $b \leq a$,

(3) $p_{a} \wedge p_{b} \rightarrow p_{a \cdot b}$ for all $a$ and $b$ in $\mathcal{B}$,

(4) $p_{b} \vee p_{1+b}$ for all $b \in \mathcal{B}$. 
Clearly, $|P|=\left|2^{\aleph_{0}}\right|$ and $\Sigma$ is a consistent set of propositions. By our hypothesis there exists a valuation $\sigma \in 2^{P}$ which satisfies $\Sigma$. It is easy to see that $\mathcal{F}=\left\{b: \sigma\left(p_{b}\right)=1\right\}$ is an ultrafilter of $\mathcal{B}$ including $H$ as required.

REMARK 7. It is known (see [8]) that in ZF:

(A) BPI iff in every Boolean algebra every filter can be extended to an ultrafilter.

However, with respect to Theorem 6, the analogy is not quite the same. We do not know whether the following implication is provable in $\mathrm{ZF}$ :

(B) Every Boolean algebra of size $\leq|\mathbb{R}|$ has an ultrafilter implies in every Boolean algebra of size $\leq|\mathbb{R}|$ every filter can be extended to an ultrafilter.

We remark here that if we assume either of the statements:

- $\mathrm{P} R$ : Each partition of $\mathbb{R}$ has size $\leq|\mathbb{R}|$ (see [11]),

- for every Boolean algebra $\mathcal{B}$ such that $|\mathcal{B}| \leq|\mathbb{R}|$ and every ideal $\mathcal{I}$ of $\mathcal{B}$, $|\mathcal{B} / \mathcal{I}| \leq|\mathbb{R}|$

then the usual proof of $(\mathrm{A})$ as given in $[17, \mathrm{p} .101]$ can be used in order to establish (B).

\section{Independence results}

TheOREM 8. None of WOAC, DC and $\mathrm{CAC}(\mathbb{R})$ implies $\operatorname{TP}\left(2^{\mathbb{R}}\right)$.

Proof. R. Solovay (see [4, p. 166]) has shown that WOAC holds in Feferman's model $\mathcal{M} 2$ in [8] (see also [3] and [9, Prob. 5.24]). Since WOAC implies DC (see [8]) and clearly $\mathrm{CAC}(\mathbb{R})$, it follows that $\mathrm{DC}$ and $\mathrm{CAC}(\mathbb{R})$ are also valid in $\mathcal{M} 2$. We shall prove that $\mathrm{TP}\left(2^{\mathbb{R}}\right)$ fails in $\mathcal{M} 2$.

Let us give a brief description of $\mathcal{M} 2$ (see, also [9, Prob. 5.24]). Let $\mathcal{M}$ be a countable transitive model of $\mathrm{ZF}+2^{\aleph_{0}}=\aleph_{1}$ and let $\mathbb{P}=\operatorname{Fn}(\omega \times \omega, 2)$ be the set of all finite partial functions $p$ from $\omega \times \omega$ into $2=\{0,1\}$ partially ordered by: $p \leq q$ iff $p \supseteq q$. Let $G$ be a $\mathbb{P}$-generic set over $\mathcal{M}$ and $\mathcal{M}[G]$ the corresponding generic extension of $\mathcal{M}$. For every $X \subset \omega \times \omega$ the function $\pi_{X}:(\mathbb{P}, \leq) \rightarrow(\mathbb{P}, \leq)$,

$$
\left(\pi_{X} p\right)((n, m))= \begin{cases}p(n, m) & \text { if }(n, m) \notin X, \\ 1-p(n, m) & \text { if }(n, m) \in X,\end{cases}
$$

is an order automorphism of $(\mathbb{P}, \leq)$, and $\mathcal{G}=\left\{\pi_{X}: X \in \mathcal{P}(\omega \times \omega)\right\}$ with the composition operation $\circ$ is a group. Furthermore, $\mathcal{E}=\left\{\operatorname{fix}(E): E \in[\omega]^{<\omega}\right\}$, where

$$
\operatorname{fix}(E)=\left\{\pi_{X}:(X \in \mathcal{P}(\omega \times \omega)) \wedge(X \cap(E \times \omega)=\emptyset)\right\},
$$

is a filterbase. Let $\mathcal{F}$ be the normal filter which is generated by $\mathcal{E}$. Then $\mathcal{M} 2$ is the corresponding symmetric model of $\mathrm{ZF}$. 
Clearly, in $\mathcal{M}[G], g=\bigcup G$ is a function from $\omega \times \omega \rightarrow 2$. For every $n \in \omega$ let $g_{n}: \omega \rightarrow 2, g_{n}(m)=g(n, m)$ and $a_{n}=g_{n}^{-1}(1)$. Clearly, both $a_{n}$ and $a_{n}^{\mathrm{c}}$ are infinite subsets of $\omega$.

For a set $a \subset \omega$ we denote by $[a]$ the set $\left\{x \triangle a: x \in[\omega]^{<\omega}\right\}$.

It is known (see [8]) that for each $n \in \omega$, the sets $a_{n}$ belong to $\mathcal{M} 2$. Hence, $a_{n}^{\mathrm{c}},\left[a_{n}\right]$, and $\left[a_{n}^{\mathrm{c}}\right]$ belong to $\mathcal{M} 2$ too. It is also known (see [8] and [9, Prob. 5.24]) that $\operatorname{UF}(\omega)$ fails in $\mathcal{M} 2$ and we have

Lemma $9([3]) . \mathcal{A}=\left\{\left\{[X],\left[X^{\mathrm{c}}\right]\right\}: X \in \mathcal{P}(\omega)\right\}$ has no choice function.

Since $\bigcup \mathcal{A}$ is clearly a partition of $\mathcal{P}(\omega)$ and $|\mathcal{P}(\omega)|=|\mathbb{R}|$ we may consider each member of $\bigcup \mathcal{A}$ as a subset of $\mathbb{R}$. Hence, we may view $\bigcup \mathcal{A}$ as a partition of $\mathbb{R}$. If $\operatorname{TP}\left(2^{\mathbb{R}}\right)$ were valid in $\mathcal{M} 2$, then by Theorem 5 (ii), $\mathcal{A}$ would have to admit a choice function, contradicting Lemma 9 . Thus, $\operatorname{TP}\left(2^{\mathbb{R}}\right)$ fails in $\mathcal{M} 2$ as claimed.

THEOREM 10.

(i) $\mathrm{AC}(\mathbb{R})$ does not imply $\mathrm{BPI}(\mathbb{R})$.

(ii) $\mathrm{BPI}(\omega)$ does not imply $\mathrm{BPI}(\mathbb{R})$. In particular, none of $\mathrm{UF}(\omega), \mathrm{UF}(\mathbb{R})$ implies $\mathrm{BPI}(\mathbb{R})$.

Proof. We shall construct a symmetric model $\mathcal{N}$ in which $\mathrm{AC}(\mathbb{R})$, hence $\mathrm{UF}(\omega)$ and $\mathrm{UF}(\mathbb{R})$ are true but $\mathrm{BPI}(\mathbb{R})$ fails. This model $\mathcal{N}$ will be the next higher cardinal analogue of Feferman's model $\mathcal{M} 2$.

Let $\mathcal{M}$ be a countable transitive model of $\mathrm{ZF}+2^{\aleph_{0}}=\aleph_{1}$. Let $\mathbb{P}=$ $\operatorname{Fn}\left(\omega \times \omega_{1}, 2, \omega_{1}\right)$ be the set of all countable partial functions $p$ from $\omega \times \omega_{1}$ into 2 partially ordered by reverse inclusion. Let $G$ be a $\mathbb{P}$-generic set over $\mathcal{M}$ and $\mathcal{M}[G]$ the corresponding generic extension of $\mathcal{M}$. As in the case of $\mathcal{M} 2$ any $X \subset \omega \times \omega_{1}$ yields an order automorphism $\pi_{X}:(\mathbb{P}, \leq) \rightarrow(\mathbb{P}, \leq),(\mathcal{G}, \circ)$, where $\mathcal{G}=\left\{\pi_{X}: X \in \mathcal{P}\left(\omega \times \omega_{1}\right)\right\}$, is a group, and $\mathcal{E}=\{\operatorname{fix}(E): E \in[\omega]<\omega\}$, where

$$
\operatorname{fix}(E)=\left\{\pi_{X}:\left(X \in \mathcal{P}\left(\omega \times \omega_{1}\right)\right) \wedge\left(X \cap\left(E \times \omega_{1}\right)=\emptyset\right)\right\},
$$

is a filterbase. Let $\mathcal{N}$ be the symmetric model of $\mathrm{ZF}$ produced by the normal filter $\mathcal{F}$ which is generated by $\mathcal{E}$. As $\mathbb{P}$ is countably closed (i.e., every countable chain $C$ of $\mathbb{P}$ has a lower bound) it is known (see [12, Theorem 6.14, p. 214]) that no new subsets of $\omega$ are added to $\mathcal{M}$. Thus, $|\mathbb{R}|=\aleph_{1}$ in $\mathcal{N}$. Hence, $\operatorname{AC}(\mathbb{R}), \operatorname{UF}(\omega), \operatorname{BPI}(\omega)$, and $\operatorname{UF}(\mathbb{R})$ are all true in $\mathcal{N}$.

Let $g=\bigcup G$ and for every $n \in \omega, a_{n}=g_{n}^{-1}(1)$, where $g_{n}: \omega_{1} \rightarrow 2$, $g_{n}(m)=g(n, m)$. Clearly $a_{n}$ as well as $a_{n}^{\mathrm{c}}$ are uncountable subsets of $\omega_{1}$.

For each set $a \subset \omega_{1}$ we denote by $[a]$ the set $\left\{x \triangle a: x \in\left[\omega_{1}\right] \leq \omega\right\}$.

Claim 1. For each $n \in \omega$, the sets $a_{n}, a_{n}^{\mathrm{c}},\left[a_{n}\right],\left[a_{n}^{\mathrm{c}}\right]$, and $\left\{\left[a_{n}\right],\left[a_{n}^{\mathrm{c}}\right]\right\}$ belong to $\mathcal{N}$. 
Proof of Claim 1. It suffices to show that $a_{n} \in \mathcal{N}$ for every $n \in \omega$. Let $\bar{a}_{n}=\left\{(\check{m}, p): m \in \omega_{1}, p \in \mathbb{P}\right.$ and $\left.p((n, m))=1\right\}$, where $\check{m}=\left\{\left(\check{y}, 1_{\mathbb{P}}\right):\right.$ $y \in m\}\left(1_{\mathbb{P}}=\emptyset\right.$ is the maximal element of $\left.\mathbb{P}\right)$ is the name of $m$ given in [12, Definition 2.10 , p. 190]. It can be readily verified that any $\pi_{X} \in \operatorname{fix}(\{n\})$ fixes $\bar{a}_{n}$ as well as each $\check{m}$ with $m \in \omega_{1}$. Thus, $\bar{a}_{n}$ is a hereditarily symmetric name for $a_{n}$ and $a_{n} \in \mathcal{N}$ as required.

Claim 2. The family $\mathcal{A}=\left\{\left\{[X],\left[X^{\mathrm{c}}\right]\right\}: X \in \mathcal{P}\left(\omega_{1}\right)\right\}$ has no choice set.

Proof of Claim 2. Assume the contrary and let $c \in \mathcal{N}$ be a choice set of $\mathcal{A}$. Let $\bar{c}, \overline{\mathcal{A}}$ be hereditarily symmetric names for $c$ and $\mathcal{A}$ respectively with $\operatorname{fix}(E) \subseteq \operatorname{sym}(\bar{c}) \cap \operatorname{sym}(\overline{\mathcal{A}})$, where for a name $\tau, \operatorname{sym}(\tau)=\{\pi \in \mathcal{G}$ : $\pi(\tau)=\tau\}$. Fix $n \in E^{c}$ and assume that $c \cap\left\{\left[a_{n}\right],\left[a_{n}^{\mathrm{c}}\right]\right\}=\left[a_{n}\right]$. Let $g \in G$ satisfy

$g \Vdash \bar{c}$ is a choice set of $\overline{\mathcal{A}}$ and $\bar{c} \cap\left\{\left(\overline{\left[a_{n}\right]}, 1_{\mathbb{P}}\right),\left(\overline{\left[a_{n}^{\mathrm{c}}\right]}, 1_{\mathbb{P}}\right)\right\}=\overline{\left[a_{n}\right]}$,

where $\overline{\left[a_{n}\right]}, \overline{\left[a_{n}^{\mathrm{c}}\right]}$ are hereditarily symmetric names for $\left[a_{n}\right]$ and $\left[a_{n}^{\mathrm{c}}\right]$ respectively. Let $X=\left(\{n\} \times \omega_{1}\right) \backslash \operatorname{dom}(g)$. Then $\pi_{X} \in \operatorname{fix}(E), \pi_{X}(\bar{c})=\bar{c}$, $\pi_{X}\left(\left\{\left(\overline{\left[a_{n}\right]}, 1_{\mathbb{P}}\right),\left(\overline{\left[a_{n}^{\mathrm{c}}\right]}, 1_{\mathbb{P}}\right)\right\}\right)=\left\{\left(\overline{\left(a_{n}\right]}, 1_{\mathbb{P}}\right),\left(\overline{\left[a_{n}^{\mathrm{c}}\right]}, 1_{\mathbb{P}}\right)\right\}$ and $\pi_{X}(g)=g$. It follows that

$g \Vdash \bar{c}$ is a choice set of $\overline{\mathcal{A}}$ and $\bar{c} \cap\left\{\left(\overline{\left[a_{n}\right]}, 1_{\mathbb{P}}\right),\left(\overline{\left[a_{n}^{\mathrm{c}}\right]}, 1_{\mathbb{P}}\right)\right\}=\pi_{X}\left(\overline{\left[a_{n}\right]}\right)$.

Since $g \in G$, it follows that $c \cap\left\{\left[a_{n}\right],\left[a_{n}^{\mathrm{c}}\right]\right\}=\operatorname{val}\left(\pi_{X}\left(\overline{\left[a_{n}\right]}\right)\right)$. It is not hard to verify that $\operatorname{val}\left(\pi_{X}\left(\overline{\left[a_{n}\right]}\right)\right)=\left[a_{n}^{\mathrm{c}}\right]$. This is a contradiction finishing the proof of Claim 2.

We now prove that $\operatorname{BPI}(\mathbb{R})$ fails in $\mathcal{N}$. Let $\mathcal{H}$ be the filter of all cocountable subsets of $\omega_{1}$, i.e., $\mathcal{H}=\left[\omega_{1}\right]$. We show that there is no ultrafilter $\mathcal{F}$ of $\mathcal{P}\left(\omega_{1}\right)$ including $\mathcal{H}$. Assume the contrary and let $\mathcal{F}$ be such an ultrafilter.

We claim that for every $X \in \mathcal{P}\left(\omega_{1}\right)$ such that $X \in\left[\omega_{1}\right]^{\omega_{1}}$ and $X^{\mathrm{c}} \in\left[\omega_{1}\right]^{\omega_{1}}$, either $[X] \subset \mathcal{F}$ or $\left[X^{\mathrm{c}}\right] \subset \mathcal{F}$. Indeed, since $\mathcal{F}$ is an ultrafilter it follows that either $X \in \mathcal{F}$ or $X^{\mathrm{c}} \in \mathcal{F}$ but not both. Assume that $X \in \mathcal{F}$. Since $\mathcal{H} \subset \mathcal{F}$ it follows that $\{X \cap H: H \in \mathcal{H}\} \subset \mathcal{F}$. Therefore, $X \backslash A \in \mathcal{F}$ for all $A \in\left[\omega_{1}\right] \leq \omega$. Thus, $X \triangle A \in \mathcal{F}$ for all $A \in\left[\omega_{1}\right] \leq \omega$ and consequently $[X] \subset \mathcal{F}$ as required. Furthermore, no member of $\left[X^{\mathrm{c}}\right]$ belongs to $\mathcal{F}$ as otherwise we would easily derive that $\emptyset \in \mathcal{F}$.

Thus, the family $\mathcal{A}$ has a choice set in $\mathcal{N}$, which is a contradiction. Hence, $\mathcal{H}$ cannot be extended to an ultrafilter of $\mathcal{P}\left(\omega_{1}\right)$ and BPI $(\mathbb{R})$ fails in $\mathcal{N}$, finishing the proof of the theorem.

Acknowledgements. I would like to thank the anonymous referee for communicating the result of Theorem 5(i) and its proof as well as the proof of $($ vi $) \rightarrow$ (i) in Theorem 6 via (vi) $\rightarrow$ (vii) $\rightarrow$ (i) which simplified my initial proof. I would also like to thank Professor P. Howard for many useful suggestions and Dr. E. Tachtsis for some remarks concerning the first draft of this paper. 


\section{References}

[1] O. De la Cruz, E. Hall, P. Howard, K. Keremedis, and J. E. Rubin, Products of compact spaces and the axiom of choice, Math. Logic Quart. 48 (2002), 508-516.

[2] O. De la Cruz, E. Hall, P. Howard, J. E. Rubin, and A. Stanley, Definitions of compactness and the axiom of choice, J. Symbolic Logic 67 (2002), 143-161.

[3] S. Feferman, Some applications of the notions of forcing and generic sets, Fund. Math. 56 (1965), 325-345.

[4] U. Felgner, Models of ZF Set Theory, Lecture Notes in Math. 223, Springer, 1971.

[5] P. R. Halmos, Lectures on Boolean Algebras, Van Nostrand, Princeton, 1963.

[6] H. Herrlich, Compactness and the Axiom of Choice, Appl. Categ. Structures 4 (1996), 1-14.

[7] P. Howard, Definitions of compact, J. Symbolic Logic 55 (1990), 645-655.

[8] P. Howard and J. E. Rubin, Consequences of the Axiom of Choice, Math. Surveys Monogr. 59, Amer. Math. Soc., Providence, RI, 1998.

[9] T. Jech, The Axiom of Choice, North-Holland, Amsterdam, 1973.

[10] K. Keremedis, The compactness of $2^{\mathbb{R}}$ and some weak forms of the axiom of choice, Math. Logic Quart. 46 (2000), 569-571.

[11] K. Keremedis and E. Tachtsis, Some weak forms of the axiom of choice restricted to the real line $\mathbb{R}$, ibid. 47 (2001), 413-422.

[12] K. Kunen, Set Theory. An Introduction to Independence Proofs, North-Holland, Amsterdam, 1983.

[13] A. Lévy, The Fraenkel-Mostowski method for independence proofs in set theory, in: J. W. Addison, G. Henkin and A. Tarski (eds.), The Theory of Models, NorthHolland, 1965, 221-228.

[14] J. Łoś and C. Ryll-Nardzewski, Effectiveness of the representation theory for Boolean algebras, Fund. Math. 41 (1954), 49-56.

[15] J. Mycielski, Two remarks on Tychonoff's product theorem, Bull. Acad. Polon. Sci. Sér. Sci. Math. Astronom. Phys. 12 (1964), 439-441.

[16] Y. Rav, Variants of Rado's selection lemma and their applications, Math. Nachr. 79 (1977), 145-165.

[17] H. Rubin and J. E. Rubin, Equivalents of the Axiom of Choice, II, North-Holland, Amsterdam, 1985.

[18] H. Rubin and D. Scott, Some topological theorems equivalent to the Boolean prime ideal theorem, Bull. Amer. Math. Soc. 60 (1954), 389.

[19] J. K. Truss, Cancellation laws for surjective cardinals, Ann. Pure Appl. Logic 27 (1984), 165-207.

[20] S. Willard, General Topology, Addison-Wesley, 1968.

Kyriakos Keremedis

Department of Mathematics

University of the Aegean

83200 Karlovassi (Samos), Greece

E-mail: kker@aegean.gr 